\title{
Voltammetric monitoring of a solid-liquid phase transition in $\mathrm{N}, \mathrm{N}, \mathrm{N}$ ',N'-tetraoctyl-2,6-diamino-9,10-anthraquinone (TODAQ)
}

\author{
Sunyhik Ahn ${ }^{1,2} \cdot$ Thomas R. Forder $^{1} \cdot$ Matthew D. Jones $^{1} \cdot$ Richard A. R. Blackburn $^{1} \cdot$ Paul S. Fordred $^{1} \cdot$ Steven D. Bull $^{1}$. \\ Frank Marken ${ }^{1}$ (D)
}

Received: 10 September 2019/Revised: 4 November 2019 / Accepted: 11 November 2019 / Published online: 25 November 2019

(C) The Author(s) 2019

\begin{abstract}
Exploratory experiments on effects from a phase transition are reported for a low-melting microcrystalline anthraquinone (N,N, $\mathrm{N}^{\prime}, \mathrm{N}^{\prime}$-tetraoctyl-2,6-diamino-9,10-anthraquinone or TODAQ). Data for the solid-liquid phase transition are obtained by differential scanning calorimetry and then compared to data obtained by voltammetry. In preliminary electrochemical measurements, microcrystal deposits on a basal plane pyrolytic graphite electrode are shown to undergo a solid-state 2-electron 2-proton reduction in contact to aqueous $0.1 \mathrm{M} \mathrm{HClO}_{4}$ with a midpoint potential $E_{\text {mid,solid }}=-0.24 \mathrm{~V}$ vs. SCE. The reduction mechanism is proposed to be limited mainly by the triple phase boundary line and some transport of TODAQ molecules towards the electrode surface for both solid and melt. A change in the apparent activation energy for this reduction is observed at $69{ }^{\circ} \mathrm{C}$, leading to an enhanced increase in reduction current with midpoint potential $E_{\text {mid,liquid }}=-0.36 \mathrm{~V}$ vs. SCE. A change of TODAQ transport along the crystal surface for solid microcrystalline material (for the solid) to diffusion within molten microdroplets (for the liquid) is proposed. Upon cooling, a transition at $60{ }^{\circ} \mathrm{C}$ back to a higher apparent activation energy is seen consistent with re-solidification of the molten phase at the electrode surface. Differential scanning calorimetry data for solid TODAQ dry and for TODAQ in contact to aqueous $0.1 \mathrm{M} \mathrm{HClO}_{4}$ confirm these transitions.
\end{abstract}

Keywords Triple phase boundary $\cdot$ Phase transition $\cdot$ Calorimetry $\cdot$ Voltammetry $\cdot$ Melting point $\cdot$ Plastic crystalline solids $\cdot$ Battery materials

\section{Introduction}

Phase transition phenomena and crystal dynamics are important in intercalation and energy storage processes, where efficient transport of charge carriers through the host materials is needed. Linking electrochemical behavior with crystal properties is therefore of wider interest, and in particular, phase transitions in solids are likely to impact on the rate of charge transport and intercalation. Melting but also more localized lattice confined transitions (e.g., increase side chain flexibility) can contribute to the charge storage behavior.

Frank Marken

f.marken@bath.ac.uk

Department of Chemistry, University of Bath, Bath BA2 7AY, UK

2 Present address: Department of Materials, University of Oxford, Parks Road, Oxford OX1 3PH, UK
The use of traditional electrochemical measurements for solid-state reactions [1] and for oil microdroplet deposits on electrode surfaces [2] have been previously reported. Solidstate voltammetry of microcrystals $[3,4]$ has been employed widely as an analysis tool for example for dyes $[5,6]$, drugs, and pesticides [7], organometallics [8], or hybrid materials [9]. Usually, an inert substrate electrode such as paraffin impregnated graphite [10] or basal plane pyrolytic graphite (bppg) [11] is employed with microcrystals immobilized at the surface, and immersed into aqueous electrolyte. For some of these systems, a triple phase boundary-based mechanism [12] has been proposed to explain propagation of the redox process over the crystal surface and/or into the solid. A similar voltammetric methodology has been developed for microdroplet deposits of water-immiscible oils at electrode surfaces [13], where the triple phase boundary reaction mechanism allowed a diverse range of liquid-liquid ion transfer processes to be studied [14] and water-immiscible oil systems to be analysed [15]. 
It is interesting to ask whether voltammetry can be employed as a tool to obtain new information about crystal dynamics or for the reversible phase transition from a microcrystal to microdroplet redox systems. Phase transitions [16-18] are commonly studied in electrochemistry, for example for electrolyte phase changes [19, 20] for electrode phase changes [21], or in smart gels [22]. It is anticipated that for the change from solid | solid microcrystal | liquid to solid | liquid microdroplet | liquid triple phase boundary reaction zone, an associated change in transport mechanism and rate occurs. Therefore, in situ phase transition information can be obtained, for example from peak current data from voltammetric experiments.
The transition for solid to liquid is the focus of this study on a novel hydrophobic microcrystalline solid, N,N,N',N'-tetraoctyl2,6-diamino-9,10-anthraquinone or TODAQ. TODAQ redox system (see Eq. 1, process 1) is employed as a model with solid-state behavior similar to "plastic crystals." The relatively low melting point is linked to the four octyl chains [23, 24]. The octyl chains effectively lower the melting point, whilst retaining $\pi-\pi$ interactions in the solid. This combination of effects imparts "plastic crystalline" properties on the solid [25]. TODAQ is a solid at room temperature with a solid-liquid phase transition readily accessible in the presence of aqueous electrolyte.

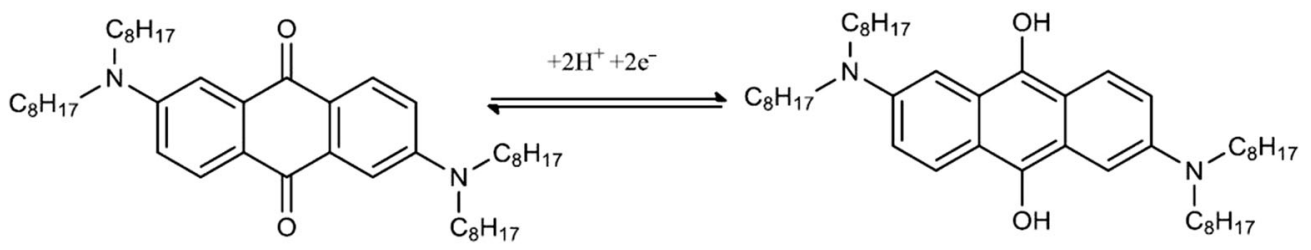

Anthraquinone and more generally quinone-type redox materials are very commonly employed for a range of applications such as solid-state intercalation cathode materials for Li-ion batteries [26, 27], homogenous catalysts for nonaqueous Li-air batteries [28, 29] aqueous redox catalysis of oxygen [30, 31]. In acidic media, a chemically reversible 2electron 2-proton reduction occurs with formation of the corresponding anthraquinol [32] (see Eq. 1, process 1).

In this study, TODAQ under acidic aqueous electrolyte conditions is therefore assumed (in first approximation) to undergo a 2-electron 2-proton reduction and back-oxidation mechanism with only minimal additional "one-electron hopping" charge transport contributions [11] to the overall transport mechanism. Here, the transport of redox active material to the electrode surface can be postulated to be dominated by both direct electron transfer at the triple phase boundary line possibly associated with some physical diffusion of molecules towards the electrode surface. This is believed to occur (i) for the soft crystalline solid via diffusion at the crystal-liquid electrolyte interfaces and (ii) for the molten liquid phase via diffusion within the melt. There should be a marked change in transport when the solid-liquid phase transition occurs. Comparison with differential scanning calorimetry (DSC) information is reported. In future, solid-liquid phase transitions based on related microcrystalline materials could be employed similarly to "smart phase-transition gels" or stimuli-response films [33] in novel sensing mechanisms (e.g., exploiting specific colligative effects on the melting point). Improved materials could be designed for proton- or cation-intercalation charge storage applications.

\section{Experimental details}

\section{Chemical reagents}

Electrolyte solutions were prepared from perchloric acid (Aldrich) and diluted to $0.1 \mathrm{M}$ with filtered and demineralised water taken from a ThermoScientific water purification system (Barnstead Nanopure) with a resistivity of not less than $18.2 \mathrm{M} \Omega \mathrm{cm}$.

\section{Instrumentation}

A microAutolab III (Metrohm) was employed in electrochemical experiments with a saturated calomel reference (Radiometer) and a platinum wire counter electrode. The working electrode was a $4.9-\mathrm{mm}$ diameter basal plane pyrolytic graphite electrode. The temperature during voltammetric experiments was controlled with a Haake thermal bath with \pm $1{ }^{\circ} \mathrm{C}$ estimated accuracy. Differential scanning calorimetry data were recorded on a TA Instruments DSC Q20 instrument.

\section{Synthesis of $N, N, N^{\prime}, N$}

'-tetraoctyl-2,6-diamino-9,10-anthraquinone (TODAQ)

Starting materials 2,6-diaminoanthraquione $(5.00 \mathrm{~g}$, $0.021 \mathrm{~mol})$ and $\mathrm{NaH}(25.20 \mathrm{~g}, 1.05 \mathrm{~mol})$ were suspended in tetrahydrofuran $(100 \mathrm{~mL})$ and 1-bromooctane $(18.13 \mathrm{~mL}$, $1.05 \mathrm{~mol}$ ) added. The resulting mixture was stirred at reflux under nitrogen for $36 \mathrm{~h}$, before being cooled to r.t. and quenched with aqueous $\mathrm{NaHCO}_{3}$. The aqueous layer was 
extracted with ethyl acetate $(2 \times 150 \mathrm{~mL})$, and the combined organics dried over $\mathrm{MgSO}_{4}$ and concentrated to give a brown oil. The crude was product was purified by column chromatography (2\% EtOAc in Hexanes) to yield the desired product as a bright orange solid $\left(5.30 \mathrm{~g}, 36 \%\right.$ yield). ${ }^{1} \mathrm{H}$ NMR $\left(400 \mathrm{MHz}, \mathrm{CDCl}_{3}\right): \delta_{\mathrm{H}}=8.09(2 \mathrm{H}, \mathrm{d}, J=9.0 \mathrm{~Hz}, \mathrm{ArH}), 7.42$ $(2 \mathrm{H}, \mathrm{d}, J=2.6 \mathrm{~Hz}, \operatorname{Ar} H), 6.80(2 \mathrm{H}, \mathrm{dd}, J=9.0,2.6 \mathrm{~Hz}, \operatorname{Ar} H)$, $3.41\left(8 \mathrm{H}, \mathrm{d}, J=7.6 \mathrm{~Hz}, \mathrm{NCH}_{2}\right), 1.69-1.59(8 \mathrm{H}, \mathrm{m}$, $\left.\mathrm{NCH}_{2} \mathrm{CH}_{2}\right) 1.41-1.20\left(40 \mathrm{H}, \mathrm{m}, \mathrm{CH}_{2}\right) 0.88(12 \mathrm{H}, \mathrm{t}, J=$ $\left.6.9 \mathrm{~Hz}, \mathrm{CH}_{3}\right) ;{ }^{13} \mathrm{C}$ NMR $\left(100 \mathrm{MHz}, \mathrm{CDCl}_{3}\right): \delta_{\mathrm{C}}=182.4$, 152.1, 136.2, 129.6, 121.6, 114.3, 108.1, 51.2, 31.8, 29.4, 29.3, 27.3, 27.1, 22.6, 14.1; IR (film, $\mathrm{cm}^{-1}$ ): $v=2953.77$, 2921.58, 2851.01, 1657.55, 1570.57, 1399.89, 1369.80, 1298.17, 1084.28, 927.91, 750.74, $719.08 \mathrm{~cm}^{-1}$.

\section{Results and discussion}

\section{Differential scanning calorimetry data for the solid-liquid phase transition for $\mathrm{N}, \mathrm{N}, \mathrm{N}^{\prime}, \mathrm{N}$ '-tetraoctyl-2,6-diamino-9,10-anthraquinone}

The study of the solid-liquid phase transition by differential scanning calorimetry (see Fig. 1b) reveals a clear phase transition at $72.2{ }^{\circ} \mathrm{C}$ (during heating) and solidification at $59.6{ }^{\circ} \mathrm{C}$ (during cooling). The heat exchanged during the phase transition is ca. $60 \mathrm{Jg}^{-1}$ corresponding to an enthalpy of fusion of $41 \mathrm{kJmol}^{-1}$, which is relatively high due to the size of the molecule. The estimated entropy of fusion is $120 \mathrm{Jmol}^{-1} \mathrm{~K}^{-1}$. These values are not very different to those for example for the hydrophobic hexadecanoic acid [34]. It is interesting to note the gradual onset of the melting process possibly indicating that parts of the structure (e.g., octyl chains) undergo melting prior to bulk melting. This kind of gradual onset is typical also for polymer materials or "plastic crystals." [35-37] When inspecting the melting process by optical microscopy (see Fig. 1a), orange plate-like crystal clearly softens and starts to merge at temperature beyond the melting point, but the changes are gradual and the crystal outline appears to be retained (or changing only very slowly).

In order to explore the effect of the aqueous electrolyte in contact to the melting microcrystals of TODAQ (and in conjunction with melting at the electrode $\mid$ aqueous electrolyte interface, vide infra), the DSC experiment was repeated with a small amount of aqueous $0.1 \mathrm{M} \mathrm{HClO}_{4}$ (ca. $50 \mathrm{wt} \%$ ) added into the sample container. Data in Fig. $1 \mathrm{~b}$ now show the effect of the aqueous phase on the phase transitions. Both melting and solidification occur at slightly lower temperatures in this case. This effect is likely to be caused by humidity and electrolyte entering the TODAQ melt (and to some extend possibly the crystal). However, both the melting and the re-
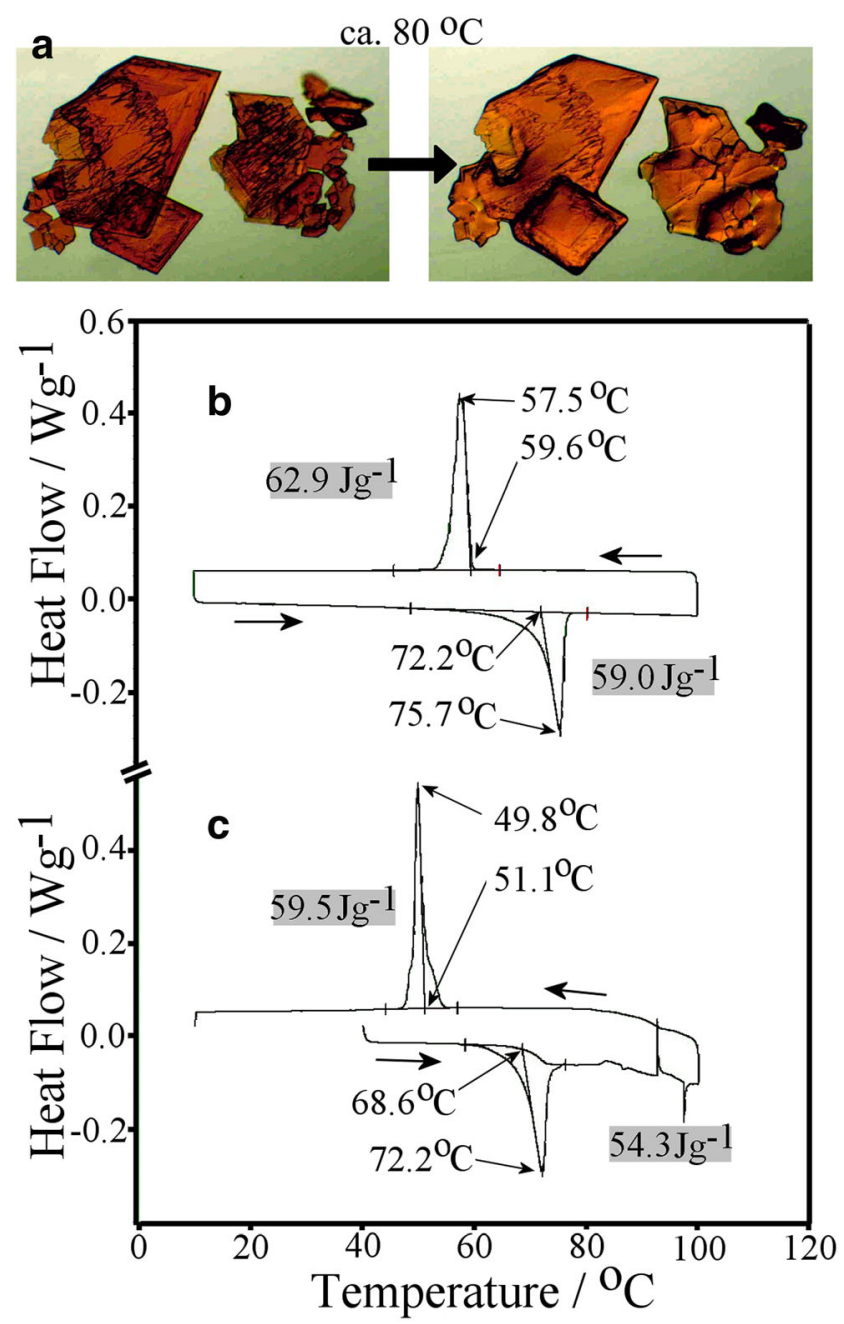

Fig. 1 a Optical micrograph of TODAQ crystals undergoing partial melting at ca. $80{ }^{\circ} \mathrm{C}$. b Differential scanning calorimetry data for TODAQ solid. c As above, but in contact to aqueous $0.1 \mathrm{M} \mathrm{HClO}_{4}$

solidification are clearly observed in the presence of aqueous electrolyte.

\section{Voltammetry data for the solid-liquid phase transition for $N, N, N^{\prime}, N$ '-tetraoctyl-1,5-diamino-9,10-anthraquinone}

Microcrystal deposits of TODAQ immobilized on a basal plane pyrolytic graphite (bppg) electrode offer an experimentally simple methodology for the solid-liquid phase transition to be investigated. TODAQ modified electrodes are immersed into aqueous $0.1 \mathrm{M} \mathrm{HClO}_{4}$ and a start potential of $+0.1 \mathrm{~V}$ vs. SCE is employed. Figure $2 \mathrm{a}$ shows weak reduction signals commencing at ca. $-0.30 \mathrm{~V}$ and a clear back-oxidation peak at $-0.05 \mathrm{~V}$ vs. SCE. The voltammetric response is consistent with only a very small fraction of the immobilized TODAQ (the charge under the oxidation peak is ca. $1 \mu \mathrm{C}$ consistent with only 5 p-mol TODAQ) being electrochemically active. The reason for the low current could be linked to only the 


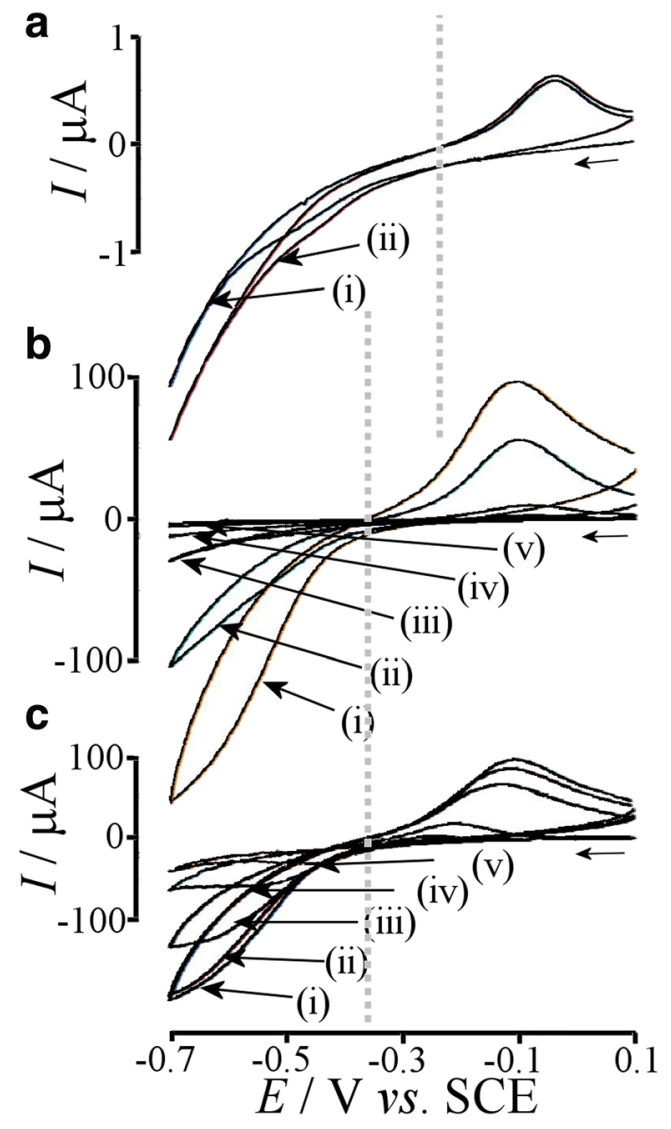

Fig. 2 a Cyclic voltammograms (scan rate $50 \mathrm{mVs}^{-1}$ ) for TODAQ immobilized on bppg and immersed in aqueous $0.1 \mathrm{M} \mathrm{HClO}_{4}$ at $30{ }^{\circ} \mathrm{C}$. Two consecutive potential cycles (i) and (ii) are shown. b Effect of temperature on the cyclic voltammogram for TODAQ at (i) 80, (ii) 70, (iii) 60, (iv) 50 , and (v) $40^{\circ} \mathrm{C}$. c Effect of scan rate on cyclic voltammograms for TODAQ at $80{ }^{\circ} \mathrm{C}$ with (i) 50 , (ii) 20 , (iii) 5 , (iv) 2 , and (v) $1 \mathrm{mVs}^{-1}$

triple phase boundary reaction zone contributing to the current response and/or only very limited transport of TODAQ molecules to the electrode surface and absence of effective electron hopping. However, when repeating the potential scan, an almost identical voltammetric signal is observed and therefore the chemical system is stable and "chemically reversible" (consistent with Eq. 1) to allow monitoring of temperature effects. The midpoint potential estimated for process 1 (see Eq. 1 ) at $30^{\circ} \mathrm{C}$ is $-0.24 \mathrm{~V}$ vs. SCE.

When increasing the temperature, the magnitude of both reduction and back-oxidation current increases (see Fig. 2b). The separation in peak potentials may in part imply slow electron transfer kinetics, but this appears to remain consistent across different temperatures. Hence, the increase in current with temperature can be attributed mainly to a substantial activation barrier in the mass transport (as observed previously for microparticle solid-state redox systems [38]). A shift in the midpoint potential is observed (see grey line in Fig. 2b) to ca. $-0.36 \mathrm{~V}$ vs. SCE. However, due to the complex shape of the voltammetric response, the midpoint potential can only be

estimated and not determined as a function of temperature. Therefore, the peak current for the back-oxidation process (as a reporter for the corresponding reduction process) is selected here as a temperature-dependent parameter. Figure $2 \mathrm{c}$ demonstrates the effect of the potential scan rate on the reduction and oxidation peak responses. At a low scan rate of less than $5 \mathrm{mVs}^{-1}$, voltammetric signals become more complicated, but for 20 or $50 \mathrm{mVs}^{-1}$, relatively stable and well-defined peaks are obtained.

Figure 3 a demonstrates the gradual change in voltammetric signal upon heating. The peak current for the oxidation peak is then plotted in an Arrhenius plot (Fig. 3b) and a clear transition (change in slope) is observed for both the heating and the cooling branches. During heating, a transition occurs at ca. $69{ }^{\circ} \mathrm{C}$ in excellent agreement with the DSC dataset (see Fig.
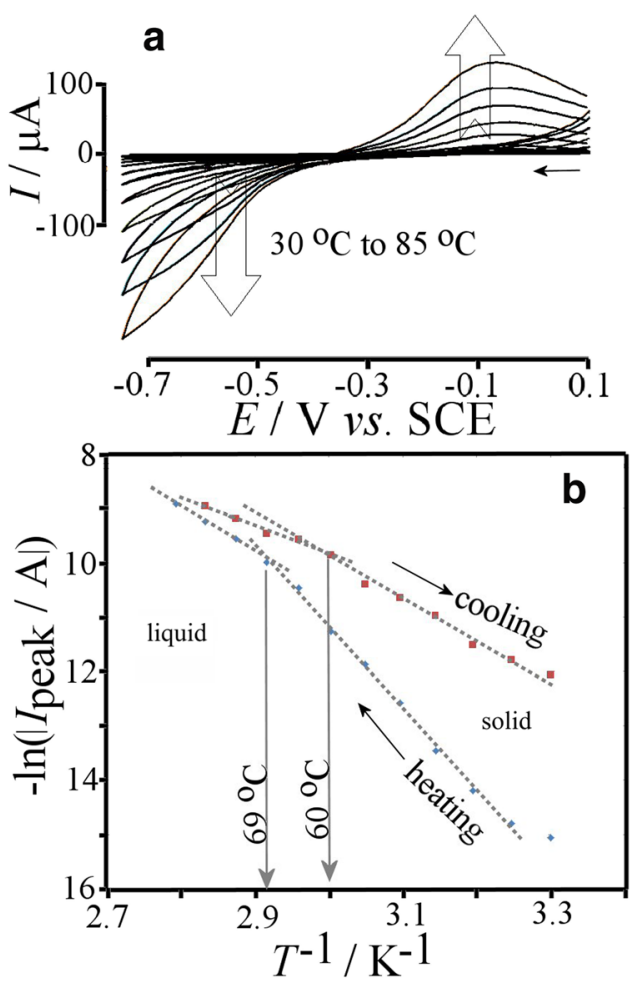

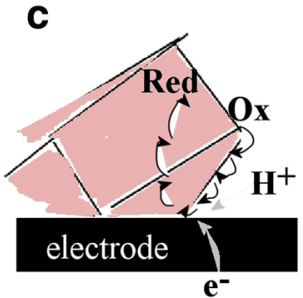

solid

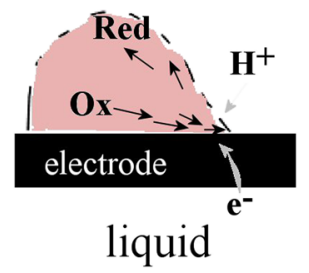

Fig. 3 a Cyclic voltammograms (scan rate $50 \mathrm{mVs}^{-1}$ ) for TODAQ immobilized on bppg and immersed in aqueous $0.1 \mathrm{M} \mathrm{HClO}_{4}$ at 30 to $85^{\circ} \mathrm{C}$ during heating in $5{ }^{\circ} \mathrm{C}$ steps. b Arrhenius plot of logarithm of absolute peak current versus inverse absolute temperature to reveal transition points in mechanism. c Schematic drawing of the overall triple phase boundary redox process for solid and liquid TODAQ 
1b). The slope of the plot in the solid and liquid regions can be used to estimate the apparent activation energy as $E_{a}=40 \pm$ $4 \mathrm{~kJ} \mathrm{~mol}^{-1}$ for the solid and $E_{a}=20 \pm 2 \mathrm{~kJ} \mathrm{~mol}^{-1}$ for the liquid (using Eq. 2). Note that the apparent activation energy for the solid appears to be very similar to the enthalpy of fusion (vide supra).These values relative to each other suggest a higher mobility after melting, but inspection of the cooling curve suggests that the absolute values are not reliable. Only the trend in apparent activation energy between solid and liquid is meaningful.

$I_{\text {peak }}=I_{\text {peak }, 0} \exp \left(\frac{-E_{a}}{R T}\right)$

In this equation, $I_{\text {peak }}$ is the peak current, $I_{\text {peak }, O}$ denotes the peak current without activation barrier (or the pre-exponential factor), $E_{a}$ is the apparent activation energy, $R$ is the gas constant, and $T$ is the absolute temperature. The separation in peak current for oxidation and reduction appears to remain consistent across different temperatures, which suggests that the process is not limited by electron transfer kinetics, but by activation energy related to mass transport. The magnitude of the observed activation energy barrier could be consistent with a diffusion process in a viscous environment (here an apparent of composite activation energy is obtained due to the peak current being probably linked to the square root of the diffusion coefficient). Upon cooling, a transition back to the solid phase is observed at ca. $60{ }^{\circ} \mathrm{C}$ (see Fig. 3b), which seems to deviate slightly from the DSC results (see Fig. 1c). However, the presence of the graphite surface and many very small microdroplets could affect the solidification process to some extent. Importantly, the hysteresis effects in the melting and solidification processes are observed directly by voltammetry.

It is interesting to note the change in Arrhenius slope in heating and cooling. The associated apparent activation barriers are lowered after re-solidification of the melted TODAQ, presumably due to a change in the solid | liquid interface between TODAQ and aqueous electrolyte. A preliminary mechanistic model for the overall process is presented schematically in Fig. 3c. For the microcrystalline solid TODAQ, it is envisaged that a surface diffusion process causes the oxidized starting material to reach the electrode surface (and the product to dissipate away from the electrode surface). Both processes appear to be very slow at room temperature. Once melting occurs, the diffusion process may occur in the bulk oil phase to give higher currents and a lower activation barrier. However, any mechanistic conclusions have to be considered preliminary, as the overall mechanism is more complex. It may be important to also consider contribution from the product, the hydroquinol, to the voltammetric wave shapes. The hydroquinol may also undergo dissolution, precipitation, and additional phase transformations. More work will be necessary to further explore these processes.

\section{Conclusion}

Melting and solidification are detected directly by voltammetry of microcrystal/ microdroplet deposits for the case of the highly hydrophobic TODAQ. For this redox system, physical transport of molecules across the crystal surface towards the electrode surface is proposed to be dominating the temperature effects on the current in the voltammetric reduction response even for the solid, thereby providing a transition in activation parameters when going from solid to molten or liquid phase. Arrhenius plot analysis of the peak current data allowed a transition point during heating to be estimated at $69{ }^{\circ} \mathrm{C}$ and solidification at $60^{\circ} \mathrm{C}$ upon cooling in close agreement with preliminary two-phase DSC data. There remain important questions to be answered about this process and, for example, the role of protonation of the amine functional groups will require further study. Also, the properties of the reduced hydroquinol product are currently unknown. Rather than employing a single working electrode, in the future, dual-plate bipotentiostatic experiments [39] could be employed to better reveal gradual changes in mass transport due to crystal transformations.

Redox systems with this type of reversible phase transition could be employed beneficially in sensing where environmental pollutants with high hydrophobicity (toluene, butylmethylether, drugs or endocrine disrupting systems, etc.) partition into the molten phase and colligatively affect the solidification process. The crystal dynamics for TODAQ and similar "plastic crystalline" redox active compounds appear of interest also with the possibility of increased mobility of molecules at temperature below those for bulk melting. Regions in the crystal with high octyl chain density may allow ingress of guest molecules (e.g., enrichment in defects). Overall, the crystal dynamics of the "plastic crystal" redox material could be highly interesting. Crystal design may help in the future to increase intercalation currents for energy storage applications. These solid-state reactions need to be investigated in more detail to be better resolved and understood in terms of molecular crystal intercalation processes.

Funding information S.A. thanks Inochem Ltd. and the Faculty of Science, University of Bath, for financial support. F.M. acknowledges financial support from the EPSRC (EP/K004956/1). T.R.F acknowledges the EPSRC (EP/G03768X/1) for funding from the DTC at Bath.

Open Access This article is distributed under the terms of the Creative Commons Attribution 4.0 International License (http:// creativecommons.org/licenses/by/4.0/), which permits unrestricted use, distribution, and reproduction in any medium, provided you give appropriate credit to the original author(s) and the source, provide a link to the Creative Commons license, and indicate if changes were made. 


\section{References}

1. Scholz F, Meyer B (1998) Electroanal Chem 20:1-86

2. Schröder U, Compton RG, Marken F, Bull SD, Davies SG, Gilmour S, Phys J (2001) Chem B 105:1344-1350

3. Scholz F, Schröder U, Gulaboski R (2005) Electrochemistry of immobilized particles and droplets. Springer, Berlin

4. Grygar T, Marken F, Schröder U, Scholz F (2002) Coll Czech Chem Commun 67:163-208

5. Domenech-Carbo A, Domenech-Carbo MT, Calisti M, Maiolo V (2010) Talanta 81:404-411

6. Bond AM, Marken F, Hill E, Compton RG, Hügel H (1997) J Chem Soc Perkin Trans 2:1735-1742

7. Domenech-Carbo A, Martini M, de Carvalho LM, Viana C, Domenech-Carbo MT, Silva M (2013) J Pharm Biomed Anal 74: 194-204

8. Bond AM, Colton R, Marken F, Walter JN (1997) Organomet 16: 5006-5014

9. Halls JE, Ahn SD, Jiang DM, Keenan LL, Burrows AD, Marken F (2013) J Electroanal Chem 689:168-175

10. Dostal A, Meyer B, Scholz F, Schröder U, Bond AM, Marken F, Shaw SJ (1995) J Phys Chem 99:2096-2103

11. Bond AM, Marken F (1994) J Electroanal Chem 372:125-135

12. Bond AM, Colton R, Daniels F, Fernando DR, Marken F, Nagaosa Y, Vansteveninck RFM, Walter JN (1993) J Am Chem Soc 115: 9556-9562

13. Marken F, Webster RD, Bull SD, Davies SG (1997) J Electroanal Chem 437:209-218

14. Banks CE, Davies TJ, Evans RG, Hignett G, Wain AJ, Lawrence NS, Wadhawan JD, Marken F, Compton RG (2003) Phys Chem Chem Phys 5:4053-4069

15. Marken F, Watkins JD, Collins AM (2011) Phys Chem Chem Phys 13:10036-10047

16. Donner C (2006) Zeitsch Physikal Chem-Inter J. Res Phys Chem Chem Phys 220:265-304

17. Levi MD, Aurbach D (1999) Electrochim Acta 45:167-185

18. Kalinin SV, Rodriguez BJ, Jesse S, Maksymovych P, Seal K, Nikiforov M, Baddorf AP, Kholkin AL, Proksch R (2008) Mater Today 11:16-27
19. Bond AM, Svestka M (1991) J Electroanal Chem 301:139-154

20. Morgenstern M, Müller J, Michely T, Comsa G (1997) Zeitsch Physik Chem- Internat J Res Phys Chem Chem Phys 198:43-72

21. Bond AM, Colton R, Harvey J, Hutton RS (1997) J Electroanal Chem 426:145-155

22. Zhou YF (2011) Prog Chem 23:125-135

23. Timmermans J, Phys J (1961) Chem Solids 18:1-8

24. Singh LP, Murthy SSN (2008) J Chem Phys 129:094501

25. Reuter D, Lunkenheimer P, Loidl A (2019) J Chem Phys 150: 244507

26. Wei W, Li L, Zhang L, Hong J, He G (2018) Electrochem Commun 90:21-25

27. Han C, Li H, Shi R, Zhang T, Tong J, Li J, Li B, Am J (2019) Chem Soc. https://doi.org/10.1039/C9TA05252F

28. Zhang P, Liu L, He X, Liu X, Wang H, He J, Zhao Y (2019) J Am Chem Soc 141:6263-6270

29. Gao X, Chen Y, Johnson L, Bruce PG (2016) Nat Mater 15:882888

30. Sarapuu A, Vaik K, Schiffrin DJ, Tammeveski K (2003) J Electroanal Chem 541:23-29

31. Kullapere M, Seinberg JM, Maeorg U, Maia G, Schiffrin DJ, Tammeveski K (2009) Electrochim Acta 54:1961-1969

32. Li Q, Batchelor-McAuley C, Lawrence NS, Hartshorne RS, Compton RG (2011) ChemPhysChem 12:1255-1257

33. Fandrich A, Buller J, Wischerhoff E, Laschewsky A, Lisdat F (2012) ChemPhysChem 13:2020-2023

34. Lide DR (1993-1994) Handbook of Chemistry and Physics, 74th edn. CRC Press, London

35. Chiu MH, Prenner EJ (2011) J Pharm Bioall Sci 3:39-59

36. Wunderlich B (2011) J Therm Anal Calorim 106:85-91

37. Wunderlich B (1999) Thermochim Acta 340-341:37-52

38. Bond AM, Colton R, Marken F, Walter JN (1994) Organometallics 13:5122-5131

39. Li M, Lewis GEM, James TD, Long YT, Kasprzyk-Hordern B, Mitchels JM, Marken F (2014) ChemElectroChem 1:1640-1646

Publisher's note Springer Nature remains neutral with regard to jurisdictional claims in published maps and institutional affiliations. 